\title{
Relación entre clima organizacional y síndrome de burnout en el servicio de emergencia de un hospital Categoría III-2. Lima, Perú 2015
}

Zenaida Solís Chuquiyauri, ${ }^{1, \mathrm{a}}$, Luisa Zamudio Eslava ${ }^{1, \mathrm{~b}}$, Juan Matzumura Kasano ${ }^{2, c}$, Hugo Gutiérrez Crespo

\section{RESUMEN}

Objetivo: Determinar la relación entre clima organizacional y Síndrome de Burnout en los profesionales de enfermería del Servicio de Emergencia de un Hospital Categoría III-2.

Material y métodos: Estudio cuantitativo, prospectivo, correlacional de corte transversal. La muestra estuvo constituida por 43 profesionales de enfermería, mediante un muestreo no probabilístico por conveniencia. Para el análisis y estudio de las variables, se utilizó la escala de Rensis Likert y Maslach Burnout Inventory.

Resultados: El clima organizacional fue nivel medio (81.4\%) seguido de un nivel alto (18.6\%) y el Síndrome de Burnout corresponde a nivel bajo (86\%) y nivel medio (14\%). La relación entre el clima organizacional y el Síndrome de Burnout fue baja (rho = -0.11).

Conclusiones: En el servicio de emergencia, el nivel de clima organizacional fue medianamente favorable con ausencia de Síndrome de Burnout en los profesionales de enfermería. No existe relación significativa entre ambas variables.

Palabras clave: agotamiento emocional, clima organizacional, síndrome de burnout.

\section{Relationship between organizational climate and burnout syndrome in the emergency service of a hospital Category III-2. Lima, Peru 2015}

\section{ABSTRACT}

Objective: To determine the relationship between organizational climate and Burnout Syndrome among nursing professionals of the Emergency Service in a Hospital Category III-2.

Material and methods: quantitative, prospective, correlational cross-sectional study. The sample consisted of 43 nurses, using a non-probability sampling for convenience. For analysis and study of the variables, Rensis Likert scale and Maslach Burnout Inventory were used.

Results: The organizational climate was average level (81.4\%) followed by a high level (18.6\%) and the Burnout Syndrome corresponds to low level (86\%) and medium (14\%) level. The relationship between organizational climate and Burnout Syndrome was low (rho $=-0.11)$.

Conclusions: In the Emergency Service, the level of organizational climate was fairly favorable with absence of Burnout Syndrome among nursing professionals. There is not significant relationship between the two variables.

Key words: emotional exhaustion, organizational climate, burnout syndrome.

1. Enfermera.

2. Médico Gineco Obstetra, profesor de la maestría en gerencia de servicios de salud.

3. Obstetra, magíster en docencia e investigación en salud.

a. Instituto Nacional de Salud del Niño San Borja. Lima, Perú.

b. Hospital Nacional Docente Madre Niño San Bartolomé.

c. Facultad de Medicina de la Universidad San Martín de Porres.

d. Coordinador académico de la Sociedad Peruana de Obstetricia y Ginecología. 


\section{INTRODUCCIÓN}

El clima organizacional y el Síndrome de Burnout, son componentes en el comportamiento cotidiano de los profesionales de salud $y$, por consecuencia, parte importante del proceso de modernización de una institución. Por otro lado, los hospitales son organizaciones públicas, donde laboran personas dentro de un espacio delimitado, atendiendo personas que en su mayoría tienen problemas de salud. Bajo esta lógica, se evidencia que los hospitales constituyen un espacio estructurado de prácticas médicas y no medicas con una adecuada coordinación, segmentación y condiciones de trabajo ${ }^{(1)}$. En estas condiciones de trabajo, alrededor del $60 \%$ de los trabajadores manifestaron problemas relacionados con el exceso de carga de trabajo y un $19 \%$ percibieron falta de apoyo social en el trabajo ${ }^{(2)}$. Otro riesgo relevante, respecto a tasas de exposición, fue al trabajo emocional, pues en algunos sectores ocupacionales como la sanidad, el $40 \%$ de los trabajadores manifestaron tener problemas relacionados con el trabajo emocional. Como consecuencia de la exposición a estos y otros tipos de riesgos, un $20 \%$ de los trabajadores en los 27 países de la Unión Europea informaron que sus condiciones de trabajo suponían un riesgo para su salud mental ${ }^{(3)}$.

Según un informe de la Organización Mundial de la Salud de 1998, a inicios del presente siglo, las enfermedades provocadas por el estrés habrían superado a las enfermedades infecciosas; también estimó que para el año 2011 en América Latina y el Caribe, se tendrían más de 88 millones de personas con trastornos afectivos desencadenados por el estrés, lo que se debería a que los estresores de la vida originan que las personas se ubiquen en una situación incómoda emocional y somáticamente, debiendo buscar la forma de cómo responder ante sus problemas $^{(4)}$.

En América Latina y El Caribe existen también varios estudios. Argentina, Brasil y Costa Rica muestran mayor nivel de Burnout, problema que afecta al 43$55 \%$ de los profesionales de enfermería y al $11 \%$ en el Perú ${ }^{(5,6)}$. En la Unidad de Cuidado Crítico del Hospital Central de la Fuerza Aérea del Perú se reportó un 15\% de niveles altos en la dimensión despersonalización; y un $20 \%$ de niveles altos en la dimensión cansancio emocional en profesionales de enfermería ${ }^{(7,8)}$. Un reciente estudio realizado por Velarde reportó que los peruanos presentan algún grado de estrés laboral, según un informe publicado por el diario El Comercio $^{(9)}$.
Dentro del equipo multidisciplinario de las instituciones de salud, la enfermería es considerada una profesión que requiere el despliegue de actividades que involucran control mental y emocional, pues implica una exposición a diferentes situaciones donde se debe preservar la vida, el cuidado al individuo, la familia y la comunidad; basándose en conocimientos científicos, técnicos y humanísticos, aplicándolos en los tres niveles de atención. Como integrantes del equipo de salud, se caracterizan por el manejo de situaciones críticas con desempeño eficiente y efectivo, de ahí que diversos autores hayan señalado que esta profesión conlleva a una gran responsabilidad, enfrentándose en forma cotidiana a presiones, conflictos y cambios continuos que conllevarían en el futuro, tras varios años de actividad laboral, a ser afectados por el Síndrome de Burnout, causando ausentismo e incapacidad $^{(10)}$.

El cuidado procedimental, expresivo y tecnológico son considerados actividades importantes que desarrolla el profesional de enfermería. Actualmente, se hace necesaria la incorporación del clima organizacional como eje básico, proporcionando retroalimentación acerca de los procesos que determinan los comportamientos organizacionales, permitiendo introducir cambios planificados tanto en las actitudes y conductas de los miembros, como en la estructura organizacional o en uno o más de los subsistemas que lo componen ${ }^{(11)}$.

El objetivo de este estudio es determinar la relación entre el clima organizacional y el Síndrome de Burnout en los profesionales de enfermería del servicio de emergencia de un Hospital Categoría III-2, durante el año 2015.

\section{MATERIAL Y MÉTODOS}

El estudio fue cuantitativo, prospectivo, correlacional de corte transversal. Con relación a la población, los profesionales con la condición de nombrados fueron $7(16 \%)$ y 37 (84\%) correspondieron a la modalidad de contrato administrativo de servicios. Como criterios de inclusión se consideraron al profesional de enfermería nombrado y por contrato administrativo de servicios, que se encontraban laborando en el servicio de emergencia, que desearon participar en el estudio. Solo un profesional fue excluido del estudio, debido a que 
realizaba actividades administrativas y no deseaba participar en el estudio. La muestra estuvo constituida por 43 profesionales de enfermería, mediante un muestreo no probabilístico.

Para el estudio de la variable Clima Organizacional, se utilizó la Escala Rensis Likert, validada por un juicio de expertos a través de la metodología de focus group. La confiabilidad correspondió a 0.73 según Alfa de Cronbach. Consta de 28 ítems que abarcan las 3 dimensiones: Cultura Organizacional, con 8 ítems (nivel bajo entre 0 a 2 puntos, medio entre 3 a 6 y alto entre 7 a 8 puntos); Diseño Organizacional, con 9 ítems (nivel bajo entre 0 a 2 puntos, medio entre 3 a 6 y alto entre 7 a 8 puntos) y Potencial Humano, con 11 ítems (nivel bajo entre 0 a 2 puntos, medio entre 3 a 8 y alto entre 9 a 11 puntos). El puntaje general del Clima Organizacional fue: nivel bajo entre 0 a 7 puntos, medio entre 7 a 21 y alto entre 22 a 28 puntos ${ }^{(11,12)}$.

Para el estudio de la variable Síndrome de Burnout, se utilizó el Inventario de Burnout de Maslach, validado por un juicio de expertos a través de la metodología de focus group. La confiabilidad correspondió a 0.73 según Alfa de Cronbach. Consta de 22 ítems que abarcan 3 dimensiones: Agotamiento Emocional, con 9 ítems (nivel bajo entre 0 a 16 puntos, nivel medio entre 17 a 26 y alto entre 27 a 54 puntos); Despersonalización, con 5 ítems (nivel bajo entre 0 a 8 puntos, medio entre 9 a 13 y alto entre 14 a 30 puntos) y Baja Realización Personal, con 8 ítems (nivel bajo entre 0 a 30 puntos, medio entre 31 a 36 y alto entre 37 a 48 puntos). El puntaje general del Síndrome de Burnout fue: nivel bajo entre 0 a 48 puntos, medio entre 49 a 83 y alto entre 84 a 132 puntos $^{(13)}$.

Los datos fueron analizados mediante estadísticas de resumen numérico y gráfico. La relación entre el Clima Organizacional y el Síndrome de Burnout fue evaluado mediante la Correlación de Spearman. Los datos fueron analizados mediante el programa SPSS, versión 23.0.

\section{RESULTADOS}

En el presente estudio se entrevistaron a 43 profesionales de enfermería. La mediana de edad fue de 35 años, la edad mínima = 28 años, edad máxima = 42 años; el $95 \%$ de sexo femenino y $90.7 \%$ con estado civil casado. De acuerdo a la condición laboral, el 86\% eran contratados y $46.5 \%$ tenían un tiempo de servicio mayor de dos años.

El clima organizacional se refiere a las características del ambiente de trabajo, percibidas directa o indirectamente por los trabajadores; los cuales repercuten en el comportamiento laboral. El clima organizacional recoge aspectos relacionados a la cultura organizacional, diseño organizacional y potencial humano.

Figura 1. Nivel de clima organizacional según sus dimensiones

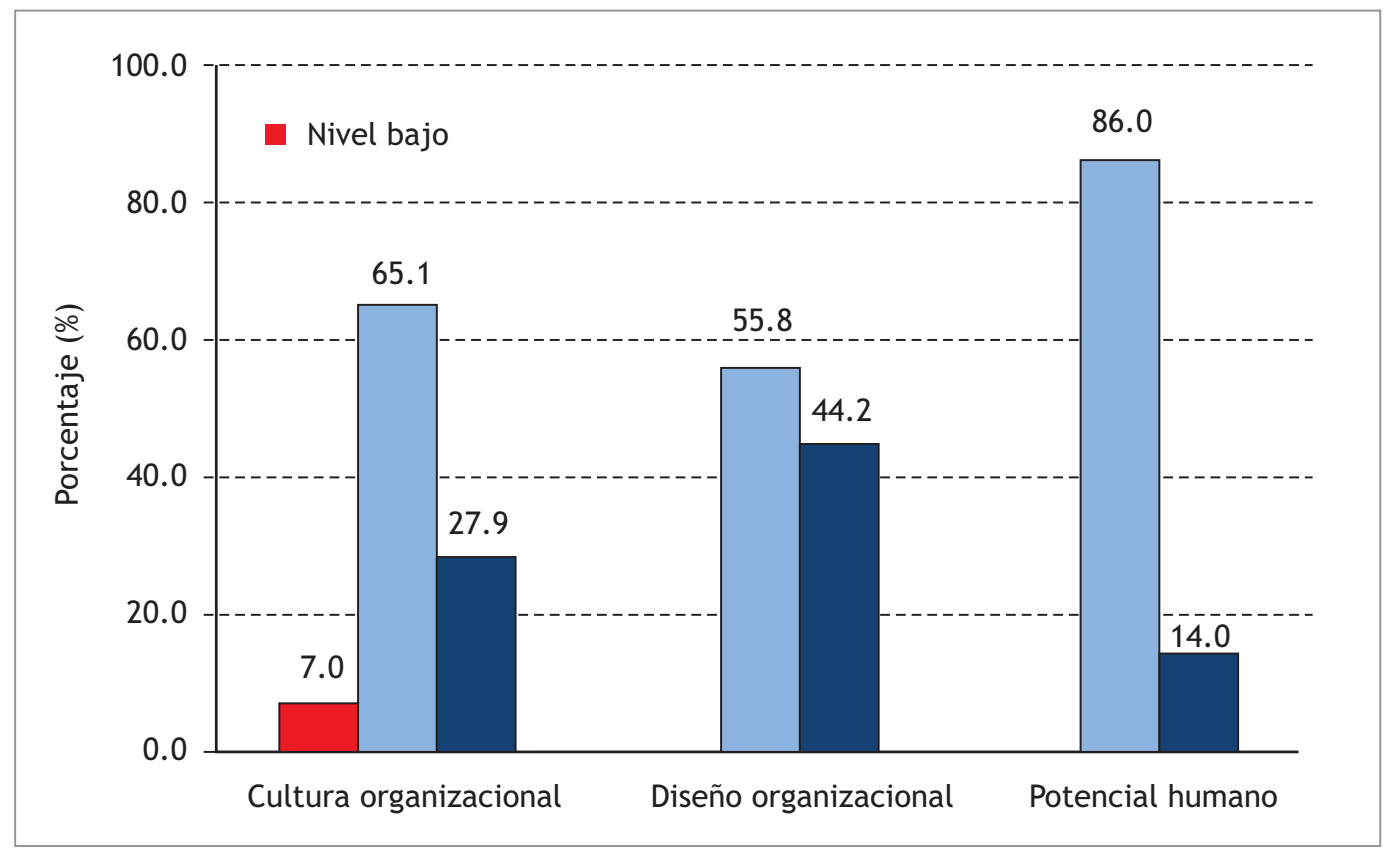


De acuerdo con los resultados obtenidos al estudiar el clima organizacional según sus dimensiones, se puede observar que la dimensión potencial humano correspondió al nivel medio en $86 \%$. La dimensión cultura organizacional al nivel medio en $65.1 \%$ y el nivel bajo 7\%. La dimensión diseño organizacional correspondía a nivel medio en $55.8 \%$ de los profesionales de enfermería. (Figura 1).

Figura 2. Puntuación final de clima organizacional por niveles

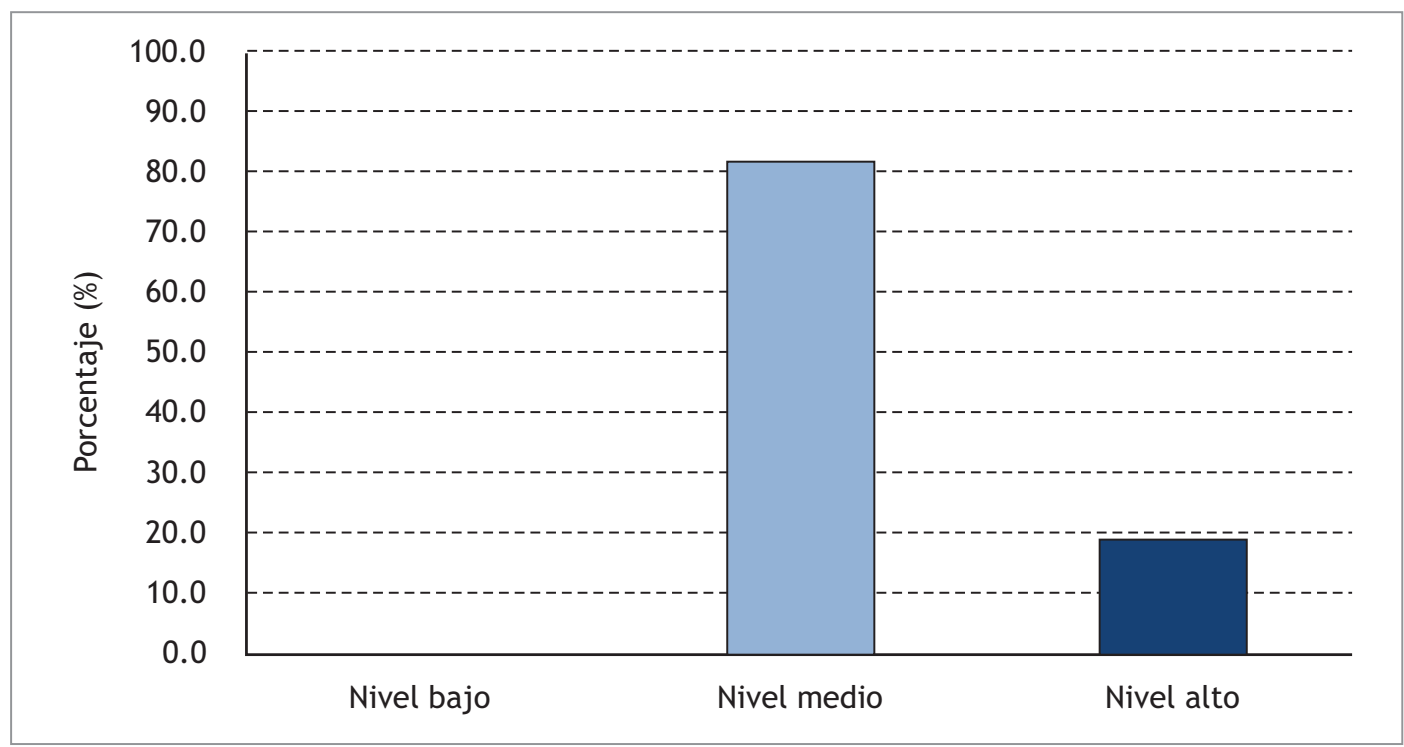

De acuerdo con los resultados la puntuación final, el clima organizacional presentó un nivel medio en $81.4 \%$ y un nivel alto en $18.6 \%$ de los profesionales de enfermería del servicio de emergencia, como se muestra en la (Figura 2).

El Síndrome de Burnout es definido como un estado de fatiga ocasionado por la falta de una recompensa esperada, tras un esfuerzo dedicado a un proyecto de vida. En la actualidad el Síndrome de Burnout es considerado como uno de los daños laborales de carácter psicosocial más importante. El Burnout es un proceso que surge como consecuencia del estrés laboral crónico, en el cual se combinan variables de carácter individual, social y organizacional.

Figura 3. Nivel de Síndrome de Burnout según sus dimensiones

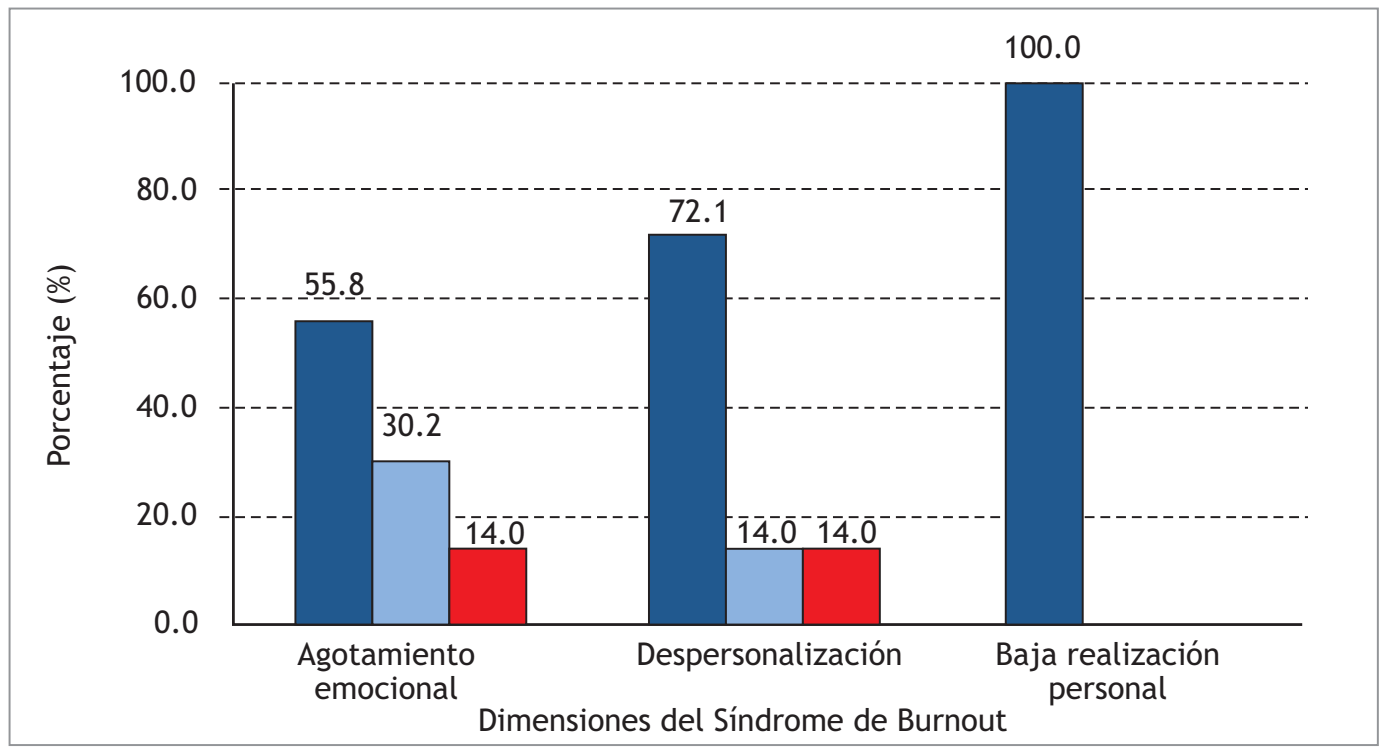


De acuerdo con las dimensiones del Síndrome de Burnout, la dimensión Baja Realización Personal fue de nivel bajo en 100\%; la dimensión Despersonalización fue nivel baja en $72.1 \%$; la dimensión Agotamiento emocional fue de nivel bajo en $55.8 \%$ seguido de un nivel medio en $30.2 \%$ para el desarrollo del Síndrome de Burnout en los profesionales de enfermería. (Figura 3).

Figura 4. Puntuación final del Síndrome de Burnout por niveles

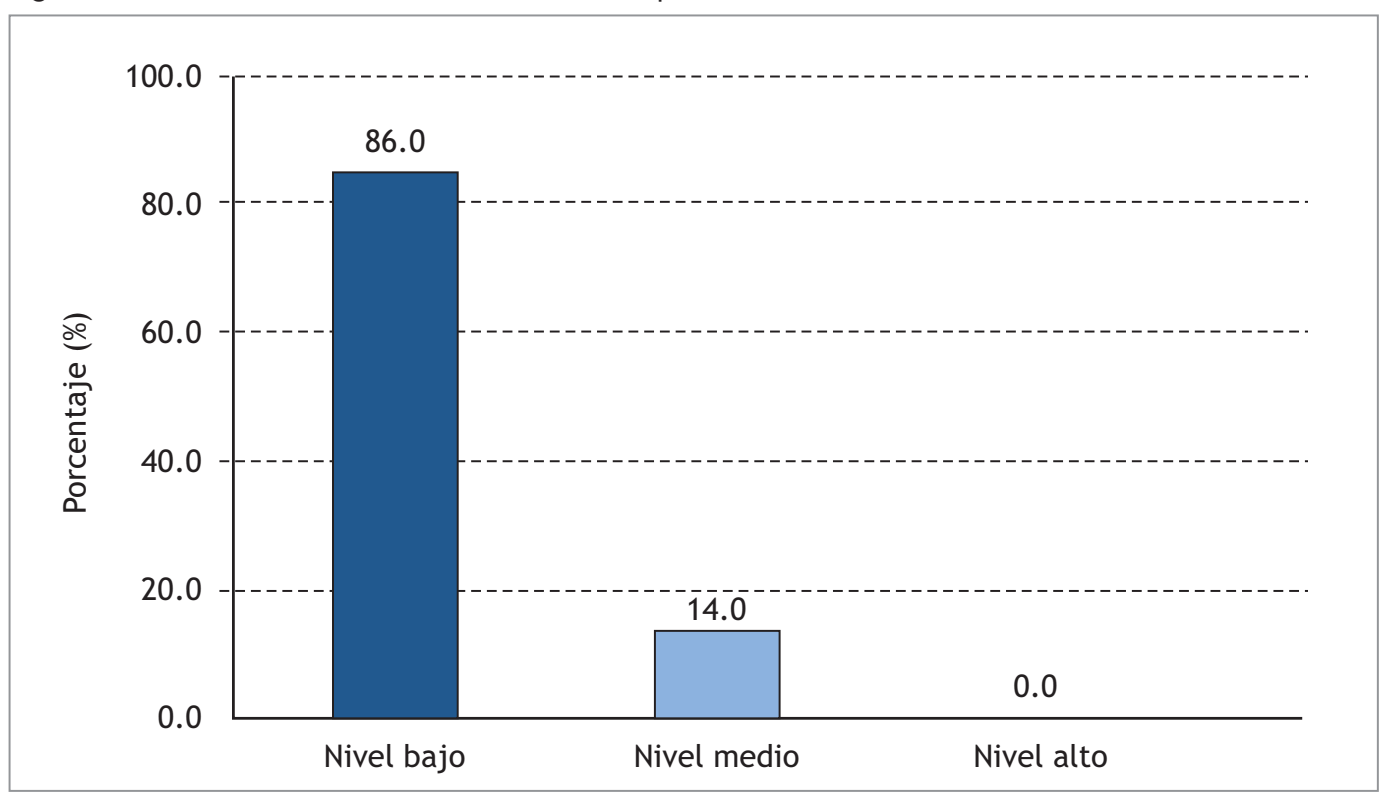

De acuerdo con la puntuación final del Síndrome de Burnout, se presentó un nivel bajo en $86 \%$, es decir, la mayoría de los profesionales de enfermería del servicio de emergencia presentaban ausencia de Síndrome de Burnout y solo un $14 \%$ un nivel medio para desarrollar el Síndrome de Burnout; (Figura 4).

Tabla 1. Relación entre el clima organizacional y el Síndrome de Burnout*

\begin{tabular}{|lcccc|}
\hline & $\begin{array}{c}\text { Síndrome } \\
\text { de Burnout }\end{array}$ & $\begin{array}{c}\text { Agotamiento } \\
\text { emocional }\end{array}$ & $\begin{array}{c}\text { Despersonalización } \\
\text { Baja realización } \\
\text { personal }\end{array}$ \\
\hline Clima organizacional & -0.11 & -0.01 & -0.31 & -0.01 \\
\hline Cultura organizacional & -0.19 & -0.12 & -0.32 & 0.01 \\
\hline Diseño organizacional & -0.06 & 0.03 & -0.31 & -0.03 \\
\hline Potencial humano & -0.06 & 0.16 & -0.06 & -0.06 \\
\hline
\end{tabular}

* Mediante Correlación de Spearman.

Con respecto al análisis de la relación según las dimensiones del clima organizacional y el Síndrome de Burnout, correspondió a una correlación negativa baja (rho < -0.4). La relación entre el clima organizacional y el Síndrome de Burnout, de acuerdo a la correlación de Spearman, fue baja (rho = -0.11); como se muestra en la (Tabla 1).

\section{DISCUSIÓN}

Los resultados del estudio muestran que la mediana de edad fue de 35 años, el 95\% fueron de sexo femenino, $90.7 \%$ de estado civil casado; el $86 \%$ son contratados y un $46.5 \%$ tenían un tiempo de servicio mayor de dos años. Estos resultados concuerdan con lo descrito por Arias y Jiménez ${ }^{(14)}$ y se debe, en parte, 
a que este grupo profesional está representado mayoritariamente por mujeres, y que en tal condición, tienen responsabilidades familiares además de las laborales, lo que aumenta aún más su vulnerabilidad.

De acuerdo con los resultados obtenidos al estudiar el clima organizacional según sus dimensiones, se puede apreciar que la dimensión potencial humano fue de nivel medio con un $86 \%$, la dimensión cultura organizacional fue de nivel medio con un $65.1 \%$ y la dimensión diseño organizacional fue de nivel medio con un $55.8 \%$ en los profesionales de enfermería, evidenciándose que existe un ambiente laboral aceptable, pero con riesgo a predisponer al Síndrome de Burnout, resultados que coinciden con los reportados por Contreras ${ }^{(15)}$, el cual refiere que los aspectos laborales que inciden en la aparición del síndrome están relacionados con el trabajo en sí mismo y con el contexto laboral, los cuales pueden actuar como desencadenantes.

Asimismo, según los resultados, la puntuación final del clima organizacional presentó un nivel medio en $81.4 \%$ y un nivel alto en $18.6 \%$ en los profesionales de enfermería del servicio de emergencia. Sin embargo, dentro de los factores que generaron mayor preocupación según las dimensiones encontramos la cultura organizacional, con un porcentaje mínimo pero significativo de $7 \%$ en nivel bajo. Esta condición hallada en nuestro estudio es similar a lo encontrado por Vizcarra ${ }^{(16)}$, quien menciona que los factores que generan mayor descontento son la falta de reconocimiento y el salario insuficiente, lo que genera un área de condición laboral desfavorable.

Con respecto a las dimensiones del Síndrome de Burnout, la dimensión baja realización personal fue de nivel bajo, en $100 \%$; la dimensión despersonalización fue de nivel baja, en $72.1 \%$; la dimensión agotamiento emocional fue de nivel bajo, en $55.8 \%$; datos que concuerdan con el estudio de Arias ${ }^{(17)}$, en donde demuestra bajos niveles de Síndrome de Burnout en la gran mayoría de trabajadores. No obstante, no deja de ser preocupante que el $30.2 \%$ de personas presenten un nivel medio para desarrollar el Síndrome de Burnout. Una posible explicación de este hallazgo es que los niveles elevados de realización personal mitigan los efectos adversos del estrés y alcanzan a proteger a los profesionales de padecer el síndrome por agotamiento emocional, o por lo menos, disminuye su probabilidad de aparición. Otra alternativa que valdría la pena estudiar, es la capacidad adaptativa de estos profesionales para hacer frente a las demandas del ambiente bajo condiciones laborales adversas.

Es importante destacar que en nuestro estudio se encontró la ausencia del Síndrome de Burnout, con un $86 \%$ y una tendencia a desarrollar Burnout en un $14 \%$, asociado a una percepción del clima organizacional medianamente favorable, resultado que concuerda con Vizcarra ${ }^{(16)}$, quien evidencia en su estudio que un $80 \%$ no presenta dicho síndrome y que un $10 \%$ presenta tendencia a desarrollar Burnout, señalando que la tendencia hace mención a dos síntomas presentados. Asimismo, el estudio de Giraldo y Martínez ${ }^{(18)}$, tiene como conclusión principal que los resultados de su investigación no evidencian la presencia del Síndrome de Burnout.

La relación entre las dimensiones del clima organizacional y las dimensiones del Síndrome de Burnout fue de correlación negativa baja (rho < -0.4). Por otro lado, la relación entre el clima organizacional y el Síndrome de Burnout, de acuerdo a la Correlación de Spearman fue baja (rho $=-0.11$ ), no existiendo relación significativa entre ambas variables. Los resultados difieren a lo que refiere Sá en su estudio ${ }^{(19)}$, cuyos resultados indican que las dimensiones de satisfacción en el trabajo, sí tienen impacto en las dimensiones del Síndrome de Burnout.

Aunque no se determinó una relación significativa entre el clima organizacional y el Síndrome de Burnout, el hecho de que exista un clima organizacional medianamente favorable y la ausencia de Síndrome de Burnout en mayor porcentaje, nos llevan a suponer que un clima organizacional óptimo es el responsable de estos niveles de Burnout. El mantener expectativas positivas frente a su labor, denotando que hay un buen nivel de rendimiento y productividad en el trabajo, permite al personal asistencial poseer el pensamiento de eficacia laboral, que conlleva a la obtención de logros importantes en su vida personal y laboral.

Después de analizar los resultados que trajeron consigo esta investigación, es evidente que las instituciones de salud del Sector Público están siendo influenciadas por la productividad y la competitividad, identificándose un escenario donde la perspectiva económica y las condiciones de mercado son imperativas.

La investigación llevada a cabo aporta un panorama sobre la realidad que subyace en algunas 
Relación entre clima organizacional y síndrome de burnout en el servicio de emergencia de un hospital Categoría III-2. Lima, Perú 2015

instituciones de salud públicas, sin embargo conviene seguir fomentando futuras investigaciones, donde se pueda ampliar la exploración de los riesgos psicosociales en el ámbito laboral afrontados por los trabajadores de estas instituciones públicas y su impacto sobre ellas.

En conclusión, el nivel del clima organizacional fue medio en $81 \%$ de los profesionales de enfermería del Servicio de Emergencia y el $86 \%$ de los profesionales del estudio presenta un nivel bajo para el Síndrome de Burnout.

No existe relación significativa entre ambas variables, predominando un clima organizacional medianamente favorable, con ausencia de Síndrome de Burnout en los profesionales de enfermería.

\section{REFERENCIAS BIBLIOGRÁFICAS}

1. Maguiña C, Galán-Rodas E. Health situation in Peru: the pending agenda. Rev Peru Med Exp Salud Pública. 2011;28(3):569-70.

2. Siebert-Adzic M. Emotions and leadership. Reasons and impact of emotions in organizational context. Work. 2012;41(1): 5671-3.

3. Parent-Thirion A, Vermeylen G, van Houten G, Lyly-Yrja na inen M, Biletta I, Cabrita J. Fifth European Working Conditions Survey. Luxemburgo: Publications Office of the European Union; 2012.

4. Lopez Ad, Murray CC. The global burden of disease, 1990-2020. Nat Med. 1998; 4(11): 1241-3.

5. Navarro MA, Camacho MA. Prevalencia del Síndrome de Burnout en los profesionales de enfermería del Hospital General Obispo Polanco en Teruel [tesis de pregrado en Internet]. Zaragoza: Escuela Universitaria de Enfermería; 2014 [acceso 15 de diciembre de 2015]. Disponible en: https: //zaguan.unizar.es/record/16253/files/TAZ-PFC-2014489.pdf

6. Organización Panamericana de la Salud. Estudio comparativo de las condiciones de trabajo y salud de los trabajadores de la salud en: Argentina, Brasil, Costa Rica y Perú [Internet]. Washington: OPS; 2012 [acceso 7 de mayo 2013]. Disponible en: http://new.paho.org/hqHSS-Cond_Trab_RHS2012.pdf

7. Ayala E. Síndrome de Burnout en el personal de enfermería de los servicios críticos del Hospital Central de la Fuerza Aérea del Perú en el año 2011 [tesis de postgrado]. Lima: Universidad Nacional Mayor de San Marcos; 2013.

8. Mera DA, More EN. Dimensiones del síndrome de Burnout entre profesionales de enfermería de áreas críticas de un hospital Minsa y Essalud de Chiclayo, 2013 [tesis de pregrado en Internet]. Chiclayo: Universidad Católica Santo Toribio de Mogrovejo; 2013 [acceso 14 de mayo 2015]. Disponible en: http://tesis.usat.edu.pe/jspui/handle/123456789/283
9. El $78 \%$ de los trabajadores peruanos confesó haber sufrido de estrés laboral. El Comercio. miércoles 16 de diciembre del 2009: economía.

10. Tapia T, Avalos M, Cesar R, Franco R, Gómez A, Rodríguez A. Síndrome de Burnout en enfermeras de un hospital regional. Rev Enferm Inst Mex Seguro Soc 2009;17(1):31-8.

11. Ministerio de Salud. Metodología para el estudio del clima organizacional. Lima: MINSA; 2009.

12. Ministerio de Salud. Plan para el estudio del clima organizacional 2008-2011. Lima: MINSA; 2009.

13. Bria M, Spânu F, Baban A, Dumitrascu DL. Maslach Burnout Inventory - General Survey: Factorial validity and invariance among Romanian healthcare professionals. Burnout Research 2014;1(3):103-11

14. Arias WL, Jiménez NA. Incidencia del síndrome de Burnout en enfermeras de los hospitales de Arequipa. Nuevos Paradigmas 2011;5(1):37-50.

15. Contreras F, Espinal L, Pachón AM, González J. Burnout, liderazgo y satisfacción laboral en el personal asistencial de un hospital de tercer nivel en Bogotá. Diversitas: Perspectivas en Psicología 2013;9(1):65-80.

16. Vizcarra MA, Llaja, V, Limo, C, Talavera J. Clima laboral, Burnout y perfil de personalidad: Un estudio en personal asistencial de un hospital público en Lima. Informes Psicológicos 2015;15(2):111-26.

17. Arias WA, Valdivia JZ. Clima organizacional, síndrome de Burnout $y$ afrontamiento en trabajadores de un Hospital Oncológico de Arequipa. Revista de Psicología 2013;15(1):3754.

18. Giraldo LF, Martínez DA. Síndrome de Burnout y su relación con algunos factores psicosociales desde la perspectiva del personal asistencial en cinco organizaciones colombianas prestadoras de servicios de salud bajo la nueva gestión pública. [Tesis de maestría]. Caldas: Universidad de Manizales; 2013.

19. Sá AMS, Martins-Silva PO, Funchal B. Burnout: o impacto da satisfação no trabalho em profissionais de enfermagem. Psicol Soc [Internet] 2014 [acceso 24 de marzo 2015]; 26(3):664- 74. Disponible en: http:/ / www.scielo.br/ pdf/psoc/v26n3/a15v26n3.pdf

Fuentes de financiamiento:

Este artículo ha sido financiado por los autores.

Conflictos de interés:

Los autores declaran no tener ningún conflicto de interés.

Correspondencia:

Zenaida Solis Chuquiyauri

Dirección: Calle Julio C. Tello º 207, El Agustino, Lima, Perú.

Teléfono: 948684913

Correo electrónico : zenaidasolisch@gmail.com

Recibido: 16 de agosto de 2016 Aprobado: 21 setiembre de 2016 